\title{
Sensitivity of Leishmania spp. to Glibenclamide and 4-Aminopiridine: A Tool for the Study of Drug Resistance Development
}

\author{
Alicia Ponte-Sucre, Yelitza Campos ${ }^{*}$, Jorge Vázquez, Heidrun Moll**, \\ Alexis Mendoza-León ${ }^{* /+}$
}

\begin{abstract}
Laboratorio de Fisiología Molecular, Instituto de Medicina Experimental, Universidad Central de Venezuela, Apartado 68256, Caracas 1062-A, Venezuela *Laboratorio de Bioquímica y Biología Molecular de Parásitos, Instituto de Biología Experimental, Universidad Central de Venezuela, Apartado 47577, Caracas 1041A, Venezuela ${ }^{* *}$ Zentrum für Infektionsforschung, University of Würzburg, Röntgenring 11, Würzburg, D-97070, Germany
\end{abstract}

We have demonstrated that Leishmania spp. grown as promastigotes, are sensitive to the $\mathrm{K}^{+}$channel inhibitors 4-aminopyridine and glibenclamide. Their host cells, the macrophages, are not affected by similar concentrations of the drugs. We have also initiated the molecular characterization of the mechanisms involved in the development of drug resistance to glibenclamide by the parasite. Therefore, we have selected experimentally and begun to characterize the Venezuelan Leishmania (Leishmania) strain, $N R$ resistant to glibenclamide [NR(Gr)]. The analysis of genomic DNA evidenced the existence of a fragment which apparently is amplified in $N R(G r)$. The fragment recognized by the pgpA probe, related to the Leishmania P-glycoprotein family and which was originally isolated from L. tarentolae, showed a size polymorfism between the sensitive and the resistant strain. These results suggest that the development of resistance to glibenclamide in the strain $N R(G r)$ might be associated with the amplification of the ltpgpA or related gene( $s$ ).

Key words: Leishmania - chemotherapy - glibenclamide - 4-aminopyridine - drug resistance - P-glycoprotein

Leishmaniasis is a disease with different clinical manifestations. The toxicity of conventional drugs such as the antimonials, and the possible development of resistance by the parasite to such compounds, emphasize the importance of the search for alternative strategies, both for its prevention and its treatment (Olliaro \& Bryceson 1993). The accurate and early diagnoses of the disease, and its effective control, are extremely important and must be adequately addressed.

From the chemotherapeutic point of view, the development and evaluation of new drugs is mainly based on their efficacy against parasites. Such activity is sometimes opposed either by the complex life cycle of the parasite or by the intracellular nature of the developmental stages of the organisms

\footnotetext{
This work was partially supported by the Venezuelan grants CDCH-UCV 09.33.0018.96, PC.09.010.94, 03.10.3750.96, 09.33.3514.95, CONICIT RPIV-110034 S1-96001411 and S1-2674 and the German Bundesministerium für Forschung and Technologie grant $\mathrm{K}$ I8906.

+Corresponding author. Fax: +58-2-753.5897

Received 11 November 1996

Accepted 11 June 1997
}

as happens in Leishmania (Olliaro \& Bryceson 1993). In this work we have attempted to evaluate the effect of 4-aminopyridine and glibenclamide, two classical inhibitors of $\mathrm{K}^{+}$channels, on the viability of Leishmania promastigotes and analyze some of the molecular mechanisms involved in the development of resistance to glibenclamide.

\section{MATERIALS AND METHODS}

Drugs - The drugs used were the classic $\mathrm{K}^{+}$ channel inhibitors 4-aminopyridine (4-AP) for voltage gated channels, and glibenclamide (GLIB) for ATP gated channels. 4-AP was dissolved in deionized water to $0.5 \mathrm{M}$ and GLIB was dissolved in ethanol to $4 \times 10^{-3} \mathrm{M}$. Both drugs were further diluted in Schneider's Drosophila medium to the desired concentrations. As GLIB has an afinity for serum albumin greater than $96 \%$, a correction has to be done to estimate the free drug concentration in the medium. Such estimation was done using the methodology described by Bennet et al. (1996). 4-AP does not have a high affinity for serum proteins as albumin thus, the free drug concentration in the medium is considered to be the same added.

Strains, cell lines and culture conditions - Table shows the different Leishmania strains used in this study. The Venezuelan strain NR was originally 
isolated in 1980 from a Venezuelan patient with tegumentary diffuse leishmaniasis and was kindly provided by Dr Angel Hernández, Instituto de Biología Celular, Universidad Central de Venezuela. Its identification was carried out in this study by RFLP analysis of the B-tubulin gene region as previously described (Mendoza-León et al. 1995).

The leishmanicidal activity of the compounds was studied in Leishmania NR promastigotes cultured in Schneider's Drosophila medium (GIBCO) supplemented with $10 \%$ fetal calf serum. The parasites were harvested at their stationary-phase of growth, the cell suspension was adjusted to a density of $2 \times 10^{6}$ cells ml-1 in a final volume of $0.1 \mathrm{ml}$ of fresh medium. Aliquots of stationary-phase Leishmania were then exposed to various concentrations of the drug (i.e., $10^{-10}-10^{-2} \mathrm{M}$ ) at room temperature for 2 or $4 \mathrm{hr}$. Subsequently, the number of live cells was estimated in each experimental vial and compared to the number of live cells of control vials incubated without drugs. The viability of Leishmania was determined by a modification of a previously described procedure (Jackson et al. 1985). At the end of the incubation time, the cells were washed twice in phosphate buffer (PBS), resuspended in the same buffer at the same density, stained for $2 \mathrm{~min}$ at $25^{\circ} \mathrm{C}$ with $125 \mu \mathrm{g} \mathrm{ml}^{-1}$ fluorescein diacetate and observed with a Nikon fluorescence microscope at $495 \mathrm{~nm}$. The results are expressed as the percentage of live parasites at each drug concentration.

The isolation of a resistant Leishmania strain was assayed in three isolates of New World Leishmania, i.e., the Venezuelan strains NR, 9012 and $\mathrm{AZV}$, all of them from the subgenus Leishmania (Table), and one from the Old World, L. (L.) major P. The strains were serially cultured at increasing concentrations of GLIB $(5 \mu \mathrm{M}$ to $100 \mu \mathrm{M})$. At each concentration step, the cells remained at least three weeks. The development of resistance was monitored comparing the growth of experimental and control cells grown in parallel.

Molecular analysis - The DNA isolation from sensitive and resistant Leishmania, their restriction analysis, as well as the DNA labelling and hybridization conditions were carried out as described by Mendoza-León et al. (1995). The pgpA probe, a genomic fragment of $800 \mathrm{bp}$ from L. tarentolae, present in the recombinant clone PM12, recognizes the P-glycoprotein (P-gp) family of Leishmania (Légaré et al. 1994). Following the digestion of the plasmid pM12 with the restriction enzyme Sac I and the fragment separation, the pgpA probe was recovered from the agarose gel and purified by the Wizard DNA purification System (Promega).

\section{RESULTS}

We have evaluated the effect of the $\mathrm{K}^{+}$channel inhibitors on stationary-phase promastigote cultures of the Venezuelan strain NR. This strain was identified as a member of the Leishmania subgenus by the polymorphism of the $\beta$-tubulin region (Fig. 1).

Previously, we have demonstrated that Leishmania strains are sensitive to $\mathrm{K}^{+}$channel inhibitors (Ponte-Sucre et al. 1995). A typical dose-response curve obtained for the viability of the strain NR is illustrated in Fig. 2. Both $\mathrm{K}^{+}$inhibitors, 4AP and GLIB, affect the viability of the promastigotes along their stationary phase of growth. The $\mathrm{LD}_{50}$ for $4-\mathrm{AP}$ was $39 \mu \mathrm{M}$ and for GLIB was even smaller, i.e., $7 \mu \mathrm{M}$. In addition, we have demonstrated that although the infection of the macrophage cell line J774 and peritoneal-exu-

TABLE

Designation of Leishmania species

\begin{tabular}{|c|c|c|c|c|}
\hline Subgenus & Species $^{a}$ & Strain designation $^{b}$ & Abbreviation & Origin \\
\hline \multicolumn{5}{|c|}{ Leishmania (L.) } \\
\hline & mexicana & MNYC/BZ/62/M379 & M379 & Belize \\
\hline & amazonensis & IFLA/BR/67/PH8 & $\mathrm{PH}_{8 \mathrm{C}}{ }^{c}$ & Brazil \\
\hline & major & MRHO/SU/59/P-strain & $\mathrm{P}$ & USSR \\
\hline & unknown $^{d}$ & & & \\
\hline & & MHOM/VE/72/AZV & AZV & Venezuela \\
\hline & & MHOM/VE/80/NR & NR & Venezuela \\
\hline & & MHOM/VE/90/LCP9012 & 9012 & Venezuela \\
\hline \multicolumn{5}{|l|}{$\operatorname{Viannia}(V)}$. \\
\hline & braziliensis & MHOM/BR/75/M2903 & M2903 & Brazil \\
\hline & guyanensis & MHOM/BR/75/M4147 & M4147 & Brazil \\
\hline
\end{tabular}

$a$ : the nomenclature of Leishmania proposed by Lainson and Shaw (1987); $b$ : the majority of these strains have been designated as reference strains by WHO; $c$ : this is a cloned derivative of $L$. (L.) amazonensis stock PH8; $d$ : these Venezuelan strains have been identified as members of Leishmania subgenus. 


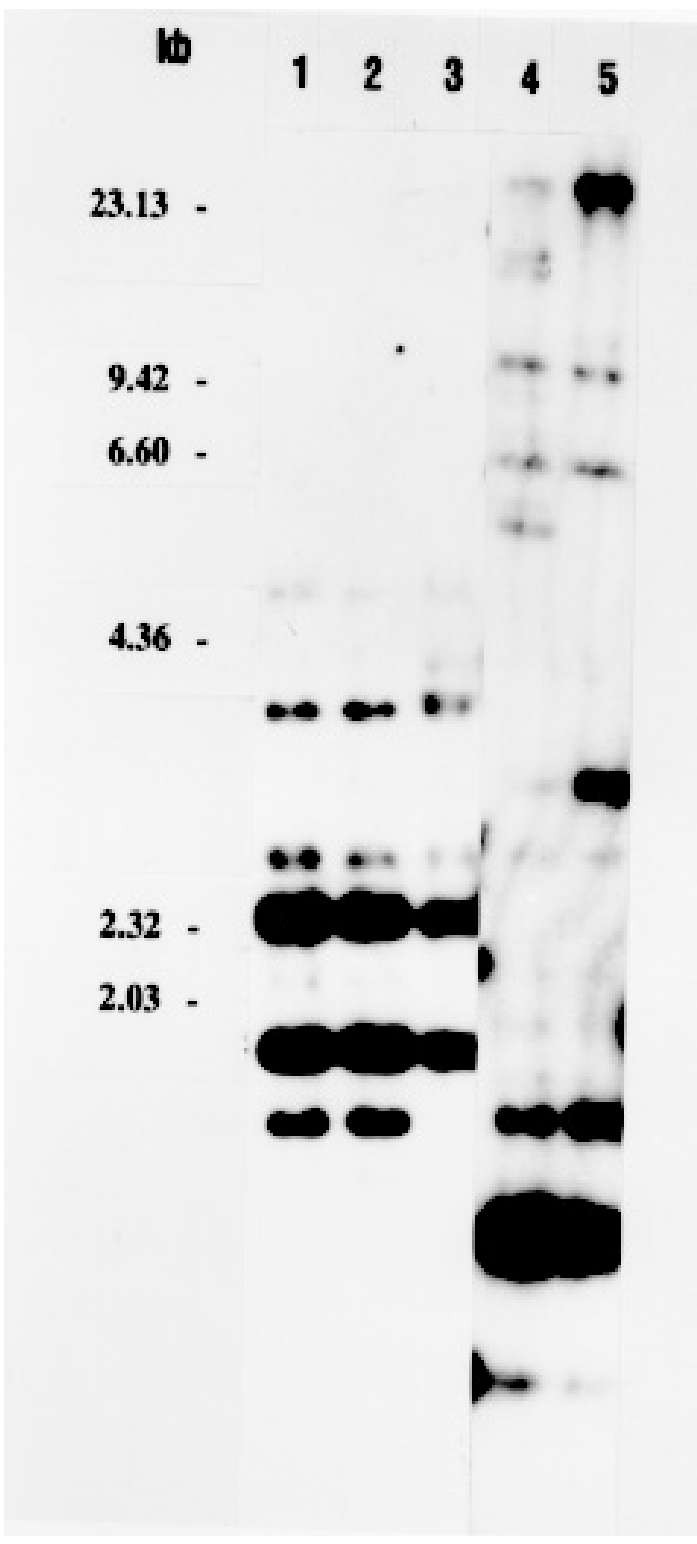

Fig. 1: Leishmania identification. Genomic DNA from New World Leishmania was digested with the endonuclease Pst I, fractionated on $1 \%$ agarose gel, and transferred to a nylon membrane. The blot was hybridized at medium stringency conditions (2x SSC, $0.1 \%$ SDS 2X Denhardt solution, $100 \mu \mathrm{g} / \mathrm{ml}$ calf thymus DNA, $67^{\circ} \mathrm{C}$ ) to the Leishmania B-tubulin clone pLgß4. L. (L.) mexicana M379 (lane 1), L. (L.) amazonensis PH8C5 (lane 2) L. (V.) braziliensis M2903 (lane 4) and L. (V.) guyanensis M4147 (lane 5), were used to identify the Venezuelan $L$. ( $L$.) NR strain (Lane 3). Molecular weight markers are from the Hind III digestions of $\lambda$-DNA. date macrophages by $L$. $(L$.) major is not significantly decreased by concentrations of the drugs 34 times smaller than the promastigote $\mathrm{LD}_{50}, 464$ $\mu \mathrm{M} 4-\mathrm{AP}$ and $10 \mu \mathrm{M}$ GLIB for this strain, the survival of intracellular parasites decreased significantly in the presence of these drugs without affecting the viability of the macrophages (data not shown). For instance, a concentration of $3 \mu \mathrm{M}$ GLIB, affects the viability of the macrophages less than $5 \%$.

In view of these results and trying to understand the response of the parasite against these drugs, we further attempted to select and molecularly characterize a Leishmania strain resistant to GLIB (Gr). Of the three isolates of New World Leishmania, NR, 9012 and AZV, and the one from the Old World $L$. (L.) major P, used for the drug resistance selection, only the strain NR survived at concentrations higher than $11 \mu \mathrm{M}(\mathrm{Gr})$; the rest of the isolates remained sensitive to the drug at concentrations higher than $8 \mu \mathrm{M}(\mathrm{Gs})$. The maximum concentration of GLIB used to select the isolate $\mathrm{NR}(\mathrm{Gr})$ was $16 \mu \mathrm{M}$. Fig. 3a illustrates the behavior of the strain NR cultured at GLIB $3 \mu \mathrm{M}$ for three consecutive weeks, and the succesful selection of the resistant strain. It can be observed that by the third week both control (Gs) and selected (Gr) cells had growing curves which were rather similar. At this stage, the parasites grown at GLIB $3 \mu \mathrm{M}$ were serially subcultured at increasing con-

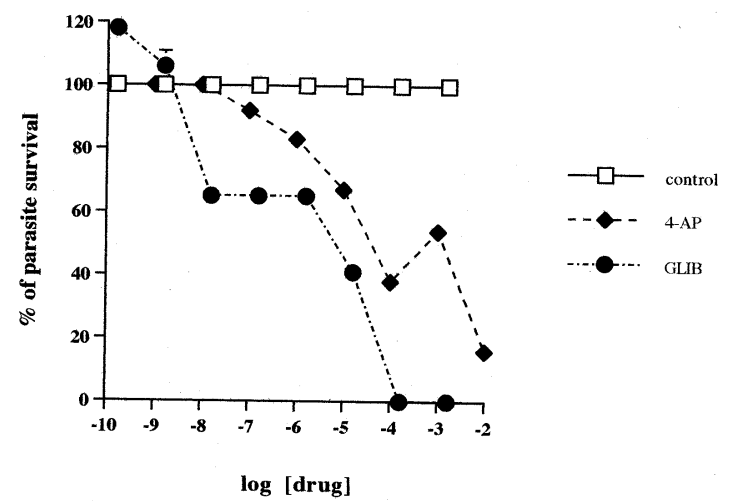

Fig. 2: effect of the ion channel inhibitors 4-aminopyridine ( ) and glibenclamide ( ) on the viability of stationary phase of the Leishmania (L.) NR strain. Data are presented as the mean \pm standard error of the mean of 6 replicates. To estimate the fraction of free drug concentration of GLIB in the medium the following equation was used: $\alpha_{\mathrm{pt}}=1 /\left[\left[\mathrm{alb}_{\mathrm{pt}} / \mathrm{alb}_{\mathrm{nl}}\right)\right]\left[\left(1-\alpha_{\mathrm{nl}}\right) /\left(\alpha_{\mathrm{nl}}\right)\right.$ +1 , where $\mathrm{alb}_{\mathrm{nl}}$ and $\alpha_{\mathrm{nl}}$ refer to values of concentration of albumin and the fraction of free drug in an standard condition and $\mathrm{alb}_{\mathrm{pt}}$ and $\alpha_{\mathrm{pt}}$ those present in the experimental medium respectively. Use of this equation assumes a molar concentration of drug far less than that of albumin, only one type of drug binding site on albumin and no cooperative binding interactions. 
centrations of the drug, up to $16 \mu \mathrm{M}$ (Fig. 3b). Fig. 3 a also demonstrates the susceptibility of the sensitive parasites to GLIB $3 \mu \mathrm{M}$ and $16 \mu \mathrm{M}$. The effect of GLIB $16 \mu \mathrm{M}$ on the NR(Gr) strain, compared to the susceptibility of the $\mathrm{NR}(\mathrm{Gs})$ strain is illustrated in Fig. 3b. These results demonstrate that the viability of the resistant strain selected in the laboratory is not impaired at this drug concentration.

An analysis of the genomic DNA of cells both of $\mathrm{NR}(\mathrm{Gs})$ and $\mathrm{NR}(\mathrm{Gr})$ was then done. DNA from both strains was digested with different restriction
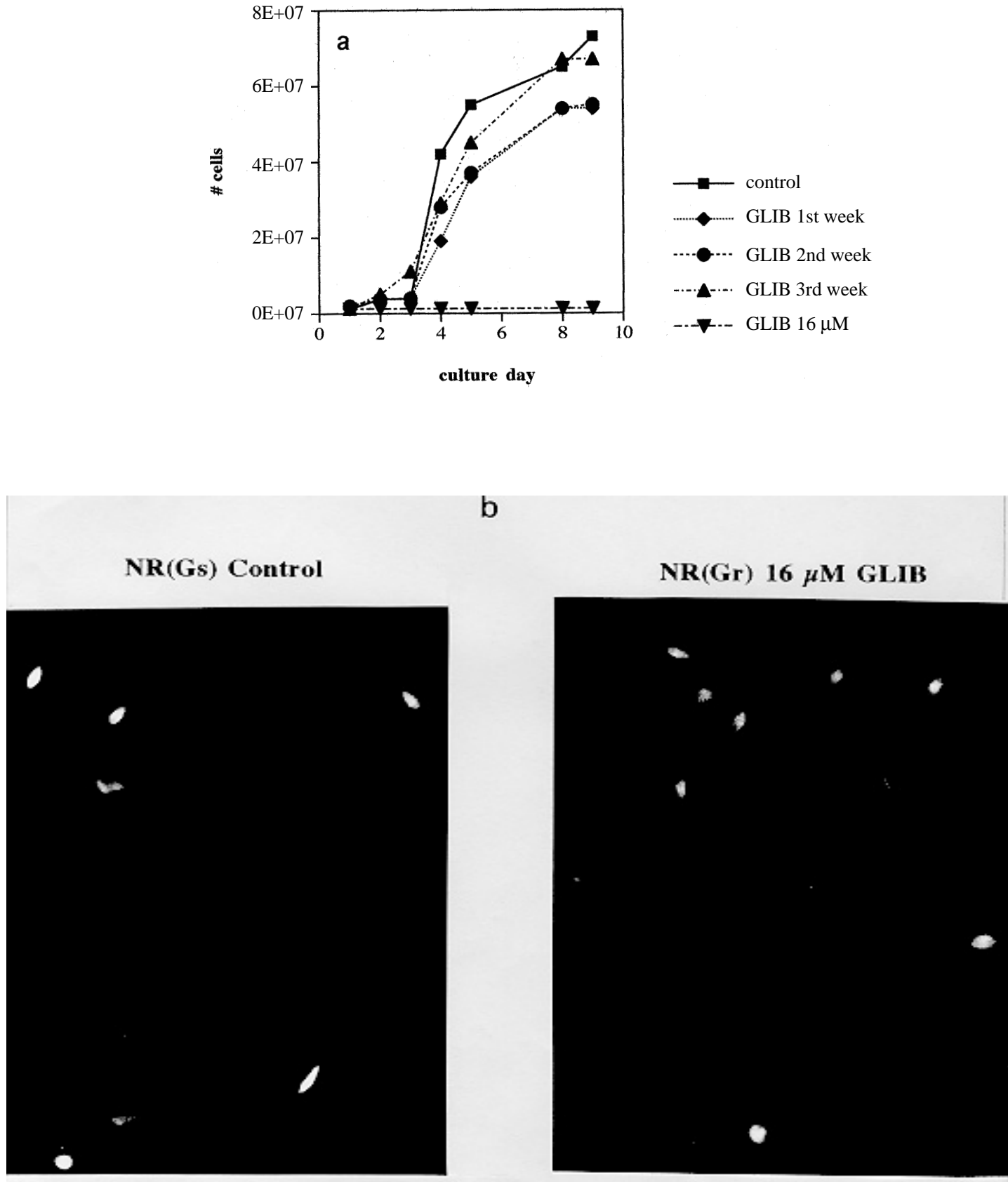

Fig. 3: sensitivity of the Venezuelan strain Leishmania (L.) NR strain (sensitive to glibenclamide) to glibenclamide $3 \mu \mathrm{M}$ ( ) and $16 \mu \mathrm{M}(\mathrm{t})$ and development of resistance by NR to GLIB $3 \mu \mathrm{M}$ at week $2(\mathrm{r})$ and $3(\mathrm{~s})$ of subculture (a) and number of alive Leishmania ( $L$.) NR cells per field in a sensitive strain NR(Gs) under control conditions and number of alive NR cells in a resistant strain NR(Gr) exposed to GLIB $16 \mu \mathrm{M}(\mathrm{b})$. Magnification $400 \mathrm{x}$. 
enzymes such as Bam HI, Pst I and Hind III, and hibridized at low Cot conditions to a pgpA probe from the Leishmania P-gp genes (related to the resistance to arsenite and antimonials). As illustrated in Fig. 4, when Pst I is used as the restriction enzyme, there is a polymorfism in the size of the fragment recognized in $\mathrm{NR}(\mathrm{Gs})(6.2 \mathrm{~Kb})$ and $\mathrm{NR}(\mathrm{Gr})(4.4 \mathrm{~Kb})$ by the probe. Since the same amount of genomic DNA was used, these results suggest that this fragment seems to be amplified in the strain $\mathrm{NR}(\mathrm{Gr})$.

\begin{tabular}{|c|c|c|c|c|c|}
\hline \multicolumn{3}{|c|}{ NR (Gs) } & \multicolumn{3}{c|}{ NR (Gs) } \\
\hline 1 & 2 & 3 & 1 & 2 & 3 \\
\hline
\end{tabular}

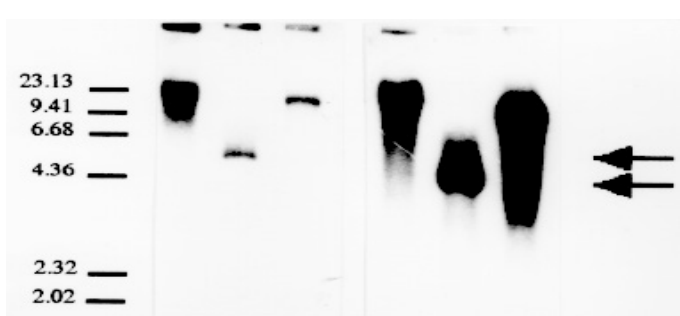

Fig. 4: southern blot analysis of pgpA sequences in Leishmania. Genomic DNA from the Venezuelan Leishmania ( $L$.) NR strain (Gs) and (Gr) was digested independently with the enzymes Bam HI (1), Pst I (2) and Hind III (3), fractionated on a $1 \%$ agarose gel and transferred to a nitrocellulose blot. The blot was hybridized to the pgpA probe under low stringency conditions. The arrows show the size polymorphism of the fragment obtained by the restriction analysis with Pst I.

\section{DISCUSSION}

The study of ion channels in parasites such as Leishmania is mainly justified by their biomedical importance (Backer-Grunwald 1992). Their complex life cycles, their flexibility and their ability to adapt to diverse environments, where the membrane plays an important role in maintaining the cellular homeostasis, also justify the study of the membrane proteins implicated on structures such as ion channels, pores and membrane transporters (Zilberstein \& Shapira 1994). The drug 4-AP has been used extensively for identifying a high variety of voltage-gated $\mathrm{K}^{+}$channels (Castle et al. 1989) and GLIB which is a $\mathrm{K}^{+}$ATP channel blocker, has a widespread use to identify $\mathrm{K}^{+}$channels associated with insulin secretion (SchmidAntomarchi et al. 1987, Panten et al. 1989). More strikingly, the sulphonylurea (GLIB-sensitive) receptor is a member of the ATP-binding cassette or traffic ATP-ase superfamily (Aguilar-Bryan et al. 1995) to which the Leishmania P-glycoprotein
(PgpA) has been associated (Légaré et al. 1994). The fact that both 4-AP and GLIB are deleterious to cultured promastigotes, have low $\mathrm{DL}_{50}$ and do not seem to impair the macrophage survival at concentrations where the survival of intracellular amastigotes decreased significantly (data not shown), reflects the importance of understanding the response and mechanism of action of these transport inhibitors. In this work we have begun to study the response of the parasite against these drugs and to acknowledge the molecular mechanisms involved in the development of GLIB resistance. For this study we have used the Venezuelan Leishmania (L.) NR strain, a member of the Leishmania subgenus. We have selected and began the characterization of Leishmania ( $L$.) NR resistant to GLIB [NR(Gr)]. Thus, we found that $\mathrm{NR}(\mathrm{Gr})$ survived at concentrations higher than $11 \mu \mathrm{M}$ as the fraction of free drug present in the medium. Its viability is not impaired at a GLIB concentration of $16 \mu \mathrm{M}$, although the viability of the sensitive strain, $\mathrm{NR}(\mathrm{Gs})$, is dramatically affected by this drug concentration. Striking was the strong signal found, independent of the restriction enzymes tested, for the pgpA in NR(Gr) in the Southern analysis. Since equal amounts of DNA was used, these results suggest an amplification of sequences related to pgpA in the GLIB resistant strain. The pgpA probe was originally characterized as the nucleotide binding site (nbs) of the gene family of P-glycoproteins in Leishmania (Ouellette et al. 1990, Légaré et al. 1994). Whether the resistance found could be related to the amplification of genes associated to Pglycoproteins is an open question. Amplification of particular gene sequences in response to drug pressure has been well documented in Leishmania; however, many of these amplifications are unrelated to drug resistance (Beverly 1991). Therefore is worthy to continue to explore the biochemical and molecular mechanisms which determine the response of the parasite to this drug and the development of resistance by this strain. On the other hand, there is a size polymorphism for the Pst I-fragment recognized by pgpA both in NR(Gs) $(6.2 \mathrm{~Kb})$ and $\mathrm{NR}(\mathrm{Gr})(4.4 \mathrm{~Kb})$. The comparison of a partial DNA sequence of the polymorphic fragment from both strains showed more than $95 \%$ of homology between them, and around 60\% homology with multidrug resistance genes from Plasmodium falciparum's (not shown). We do not have yet an explanation for it; nevertheless, internal gene rearrangements has been postulated as one mechanism where by it could arise; to answer this question we are currently working on the restriction map and final DNA sequence of the fragment from both strains. 


\section{ACKNOWLEDGMENTS}

To Dr M Ouellette for the generous gift of the pgpA probe.

\section{REFERENCES}

Aguilar-Bryan L, Nichols CG, Wechsler SW, Clemant IV JP, Boyd III E, González G, Herrera-Sosa H, Nguy K, Bryan J, Nelson D 1995. Cloning of the ßcell high-affinity sulphonylurea receptor: A regulator of insulin secretion. Science 268: 423-426.

Bakker-Grunwald T 1992. Ion transport in parasitic protozoa. J Exp Biol 172: 311-322.

Bennet L, Oie S, Schwartz JB 1996. Design and optimization of dosage regimens; pharmacokinetic data, $\mathrm{p}$. 1707-1711. In L Goodman, A Gilman (eds). The Pharmacological Basis of Therapeutics, 9th ed., McGraw Hill, New York.

Beverly S 1991. Gene amplification in Leishmania. Ann Rev Microbiol 45: 417-444.

Castle N, Haylett D, Jenkinson D 1989. Toxins in the characterization of potassium channels. Trends in Neuroscience 12: 59-65.

Jackson P, Pappas M, Hansen B 1985. Fluorogenic substrate detection of viable intracellular and extracellular pathogenic protozoa. Science 227: 435-438.

Lainson R, Shaw JJ 1987. Evolution, classification and geographical distribution, p. 1-120. In W Peters, R Killick-Kendrick (eds). The Leishmaniases in Biology and Medicine, Academic Press, London.

Légaré D, Hettema E, Ouellette M 1994. The P-glyco- protein-related gene family in Leishmania. Mol Biochem Parasitol 68: 81-94.

Mendoza-León A, Havercroft J, Barker D 1995. The RFLP analysis of the $\beta$-tubulin gene region in New World Leishmania. Parasitology 111: 1-9.

Olliaro PL, Bryceson A 1993. Practical progress and new drugs for changing patterns of leishmaniasis. Parasitol Today 9: 323-328.

Ouellette M, Fase-Fowler F, Borst P 1990. The amplified $\mathrm{H}$ circle of methotrexate-resistant Leishmania tarentolae contains a novel P-glycoprotein gene. EMBO J 9: 1027-1033.

Panten U, Burgfeld J, Goerke F, Rennicke M, Schwanstecher M, Walasch A, Zücker B, Lenzen S 1989. Control of insulin secretion by sulphonylureas, meglitinide and diazoxide in relation to their binding to the sulphonylurea receptor in pancreatic islets. Bioch Pharmacol 38: 1217-1229.

Ponte-Sucre AI, Fernández M, Mendoza-León A 1995. The effect of ionic channel inhibitors on the growth and viability of Leishmania subgenus. Abstract 345 . Molecular Parasitology Meeting, Woods Hole, Mass., USA.

Schmid-Antomarchi H, Deweile J, Fosset M Lazdunski M 1987. The receptor for the antidiabetic sulphonylureas controls the activity of the ATPmodulated $\mathrm{K}^{+}$channel. J B Chem 262: 15840-15844.

Zilberstein D, Shapira M 1994. The role of $\mathrm{pH}$ and temperature in the development of Leishmania parasites. Annu Rev Microbiol 48: 449-470. 